\title{
La masculinidad como máscara: clase, género y sexualidad en las masculinidades adolescentes
}

\author{
Masculinity as a mask: class, gender and sexuality \\ in youth masculinities
}

\author{
Mar Venegas (D) http://orcid.org/0000-0001-7500-8005 \\ Universidad de Granada, España, mariter@ugr.es
}

\begin{abstract}
The study of masculinities holds a prominent place in Feminist and Gender Studies nowadays. This paper presents an analysis based on indepth personal interviews from three case studies in Compulsory Secondary Education, with the aim of analyzing adolescent masculinities (12-18 years of age) in a city in southern Spain and their representation of sexuality, understood as a "mask" that is constructed through gender socialization and subjectivation processes to later on identify the class and gender marks that define these two processes. The results reveal a conflict between the dominant social norm regarding sexuality, internalized through gender socialization, and resistance to such norm, activating gender subjectivation. The resolution of this conflict describes the process of construction of the mask that adolescent masculinity is, in which the marks of social class become evident as well.
\end{abstract}

Key words: masculinity, youth, sexuality, gender, social class.

Resumen: El estudio de las masculinidades ocupa un lugar destacado en los estudios feministas y de género actualmente. Este artículo presenta un análisis basado en entrevistas personales en profundidad, procedentes de tres estudios de caso en educación secundaria obligatoria, con el objetivo de analizar las masculinidades adolescentes (12-18 años) en una ciudad del sur de España y su representación de la sexualidad, entendida como "máscara" que se construye a través de los procesos de socialización y subjetivación de género, para identificar las marcas de clase y género que definen esos procesos. Los resultados revelan un conflicto entre la norma social dominante sobre sexualidad, internalizada a través de la socialización de género, y la resistencia a esa norma, que activa la subjetivación de género. La resolución de ese conflicto describe el proceso de construcción de la máscara que es la masculinidad adolescente, en donde están presentes

Recepción:

$18 / 03 / 20$

Aprobación:

$24 / 06 / 20$ también las marcas de clase.

Palabras clave: masculinidad, adolescencia, sexualidad, género, clase social. 


\section{Introducción}

En los estudios sociales, el primer acercamiento a la mujer (en singular) como objeto de investigación carecía del enfoque feminista que se fue adoptando con posterioridad. Más tarde, la teoría feminista pasó a considerar a las mujeres (en plural), para visibilizar su heterogeneidad. Posteriormente, los estudios de género introdujeron las relaciones de género como unidad de análisis, al comprender que la desigualdad y opresión sufrida históricamente por las mujeres sólo puede entenderse en el marco de las relaciones entre mujeres y hombres (Narotzky, 1995; Moore, 1999). Así,

la importancia de los estudios de masculinidad radica en su capacidad para analizar las prácticas y representaciones de los varones desde sus especificidades de género, siendo dichas prácticas parte de unas relaciones sociales que colocan a los hombres mayoritariamente en una posición de dominación (Gómez et al., 2016: 156).

Desde dicho enfoque de género, este artículo se centra en un fenómeno de interés en el estudio de la masculinidad y la sexualidad en la adolescencia, que Haste (2013: 525) delimita acertadamente:

al centrarse en el comportamiento problemático de los chicos, existe el peligro de perder oportunidades para tener discusiones constructivas sobre sexo y de pasar por alto el hecho de que incluso los alumnos ruidosos y perturbadores tienen una gran cantidad de preguntas, valores y preocupaciones que se van cociendo a fuego lento "justo debajo de la superficie".

La masculinidad es así una máscara. De ahí la necesidad de observar lo que subyace justo bajo esa superficie, a cuyo análisis se dedica este artículo. Para ello, se seguirá la teoría de la construcción social del sujeto - masculino, en este caso- a través de los procesos de socialización y subjetivación ${ }^{1}$-sexual, en este caso-, atendiendo a dos principios de estructuración social de especial relevancia como son la clase social y el género.

Dos consideraciones de partida delimitan el espacio generizado de las masculinidades adolescentes que son objeto de análisis en este artículo: por un lado, la posición socioestructural privilegiada de las masculinidades; y por otro, que esa superficie de privilegio es una máscara bajo la cual subyacen el conflicto y la contradicción que suponen las normas sociales de género. En la mayoría de los estudios disponibles, la construcción de las masculinidades se entiende como un proceso de aprendizaje de la norma social de género (Harris, 1995), donde las representaciones sobre sexualidad pueden ser consideradas un constructo fruto de la "masculinidad

1 Para un conocimiento detallado de esta teoría y sus referentes teóricos y epistemológicos en el ámbito de las ciencias sociales, véase Venegas (2017). 
hegemónica" (Connell, 1987); esto es, una máscara que oculta lo que subyace "justo debajo de la superficie", como indica Haste parafraseando a Blake (2004: 281, citado en Haste, 2013: 523).

Esta propuesta constituye la tesis central del presente artículo. Así, partiendo de estas consideraciones, el objetivo es analizar precisamente eso que subyace tras la máscara, las masculinidades adolescentes, en plural, pues son dinámicas, múltiples, cambiantes, diversas. Para ello, el artículo se centra en el análisis de la representación que se hace de la sexualidad, atendiendo a su contexto social de referencia (clase social), para conocer los procesos de socialización y subjetivación (Venegas, 2017) que subyacen a la construcción de esa máscara que es la masculinidad y que en este estudio se concreta en la etapa vital de la adolescencia (12-18 años).

El análisis de la masculinidad como "máscara" es de especial importancia para la investigación en ciencias sociales en la actualidad, en particular en el marco de un modelo comprehensivo de educación afectivosexual (Ketting e Ivanova, 2018), holístico y democrático, orientado a la promoción de la igualdad sexual y de género, y, por ende, de la justicia social (Venegas, 2011c). Y ello porque la masculinidad adolescente construida sobre un modelo patriarcal (Willis, 1988 y 2003; Connell y Messerschmidt, 2005; Bourdieu, 2005; Forrest, 2000; Richardson, 2010) se ve confrontada por una masculinidad inclusiva (Anderson y McCormack, 2018), sobre la que es necesario edificar las políticas educativas y sociales que apuesten por sostener la democracia sobre esos pilares de igualdad y justicia social.

\section{Las masculinidades adolescentes en el marco de los estudios de género y sexualidad}

En este trabajo se define "género" como categoría de análisis (Esteban, 2001; Maquieira, 2001) y principio de estructuración social (Harding, 1986; Thurén, 1993), así como construcción social, histórica y cultural, no esencia universal, fija e inamovible, pues se defiende que la práctica puede rehacer continuamente la estructura social (Connell, 1987). El género contiene las dos dimensiones que conforman la realidad social: material y simbólica (Thurén, 1993; Moore, 1999). Es transversal a toda la sociedad.

Las relaciones sociales de género son consideradas como mediadas por el poder (Connell, 1987; Thurén, 1993; Maquieira, 2001), que es el principio que genera la desigualdad entre las diversas categorías de género, dando lugar a la estructuración del patriarcado como modo de dominación de género. De ello deriva la importancia del parentesco en la emergencia de la opresión de 
género y su reproducción social (Rubin, 1975; Thurén, 1993; Weeks, 2003). Un análisis comprehensivo del género implica su imbricación con otras formas de desigualdad estructural -clase social, orientación sexual, pertenencia étnica, etcétera-; esto es, la interseccionalidad (Crenshaw, 1989; Verloo, 2006).

Sobre la base de dichos principios, este artículo sigue la teoría del devenir sujeto -sexual y generizado-, mediante los dos procesos complementarios que son socialización y subjetivación (que ya fue desarrollada en otro lugar, véase: Venegas, 2017), según la cual el individuo deviene sujeto mediante un mecanismo de poder que se despliega en un proceso doble y simultáneo: estar sujeto a y ser sujeto de (Butler, 1997). Por estar sometido a discursos, sobre género y sexualidad dominantes, el individuo deviene sujeto normalizado en un modelo social dominante. Por ser sujeto de prácticas, el individuo deviene agente o sujeto de la acción social, una acción que se dilucida en el debate continuo entre la constricción y la posibilidad (Touraine, 2005),

en tanto que la acción estructuralmente situada es una acción relacional y, por ende, mediada por el poder, que es tanto externo al sujeto como su propia jurisdicción.

De este modo, acción, sujeto y estructura quedan interconectadas en procesos de mantenimiento de las estructuras sociales, a través de la socialización, y la consecuente sumisión, que conduce al sujeto a adherirse a ese orden estructural; pero también de desafío a esas estructuras sociales, mediante la subjetivación que hace posible la resistencia, entendida como contestación, que activa, a través de la reflexividad de la práctica, la posibilidad de cambio social (Venegas, 2017:31-32).

Así pues, esta teoría refleja el interés de los estudios de género y sexualidad por revisitar las teorías de género, para incorporar el análisis crítico de las masculinidades en la necesidad de comprender a éstas en su complejidad, en su producción y reproducción, pero también en los procesos de cambio social en que están implicados los hombres en pro de la igualdad, la democracia y la justicia social. En esta línea, destaca el trabajo de Harris (1995) sobre los "mensajes que escuchan los hombres", siendo esos mensajes las normas sociales de género que, según él, comprenden tres tipos de mensaje para construir la máscara que es la masculinidad:

- Mensajes para aprender a ser un hombre: la construcción de la identidad de género.

- Mensajes para actuar como un hombre: las prácticas de género.

- Constatación de las diferencias entre hombres: diversidad de modelos de género, es decir, la existencia de masculinidades, en plural. 
$\mathrm{El}$ autor concluye que "el patriarcado [...] promueve normas que los hombres aprenden a valorar para promover un orden social que beneficia a unos cuantos hombres, mientras oprime a muchos otros"2 (Harris, 1995: 190). Este principio diferenciador interno a la categoría "hombres" ya había sido señalado por Connell (1987). De ahí el interés de los grupos de hombres por promover un cambio social hacia la igualdad efectiva de derechos y oportunidades entre mujeres y hombres, a ambos niveles inter e intragrupales, dando lugar al intento por establecer un movimiento de "liberación de los hombres" en la década de 1970 (Connell, 1997), que coincide con el movimiento de liberación de las mujeres (Segunda Ola Feminista), compartiendo su inspiración feminista. Sin embargo, como indica Connell (1997), los movimientos de liberación son fruto de la opresión que sufren sus miembros y de su lucha por ponerle fin. Como grupo social, los hombres, especialmente los heterosexuales que representan la "masculinidad hegemónica" (Connell, 1987; Connell y Messerschmidt, 2005), no son un grupo oprimido. Sólo los intereses compartidos por mujeres y hombres pueden conducir a la lucha por la justicia social.

Ahí radica el enfoque relacional del género antes mencionado. Para Connell y Messerschmidt (2005), la masculinidad hegemónica es un modelo de prácticas que permite a los hombres que la dominación sobre las mujeres continúe. Difiere de otras masculinidades, sobre todo de las subordinadas. Sólo un pequeño grupo de hombres puede ejercerla. Los hombres que reciben los beneficios del patriarcado sin propagar una versión fuerte de la dominación masculina muestran una masculinidad cómplice. Hegemonía significa aquí condición alcanzada a través de la cultura, las instituciones y la persuasión; por tanto, los órdenes de género construyen masculinidades múltiples. Esa diversidad de modelos, que tanto Harris (1995) como Connell y Messerschmidt (2005) explican, sirve de referencia en los procesos de subjetivación sexual y de género de los hombres, la cual, como se señalaba antes, puede activar la resistencia de los sujetos y con ello un potencial cambio social.

En línea con estos planteamientos, Bourdieu (2005) analiza la "dominación masculina” en clave de la relación entre virilidad y violencia, que localiza en la propia socialización de género, y coincide con Connell (1987 y 1997) y Harris (1995) en explicar que "la virilidad [...] es fundamentalmente una carga" (Bourdieu, 2005: 68), una máscara, y que "la virilidad es un concepto eminentemente relacional, construido ante y para los restantes hombres

2 La traducción de todos los originales en inglés fue realizada por quien escribe este artículo. 
y contra la feminidad, en una especie de miedo a lo femenino" (Bourdieu, 2005: 71). Bourdieu (2005) examina la masculinidad también como "nobleza", observando que hay una oposición público/masculino versus privado/femenino a modo de disposiciones permanentes con un efecto Pigmalión, de manera que "basta con que los hombres se apoderen de las tareas consideradas femeninas y las realicen fuera de la esfera privada para que se vean ennoblecidas y transfiguradas" (Bourdieu, 2005: 79).

Se va perfilando así un campo de estudio de las masculinidades, desde una perspectiva feminista y crítica, que pretende cuestionar la normatividad prescriptiva del género. Se reconoce que la norma de género, que es la masculinidad, impone una máscara sobre los sujetos masculinos provocando desasosiego, como lo hace la feminidad sobre los sujetos femeninos, si bien, los hombres son la categoría social privilegiada en la estructuración de la sociedad en función del género, reconociéndose asimismo diversidad de posiciones y modelos internos a esa categoría social "hombres". El modelo de masculinidad patriarcal, desigualitaria, hegemónica, dominante, es el centro de estos análisis. No será hasta fechas recientes cuando se comience a hablar de masculinidades inclusivas (Anderson y McCormack, 2018).

Por su parte, el estudio de las masculinidades adolescentes se ha desarrollado especialmente en el ámbito de la educación (Haywood y Mac an Ghaill, 2012), donde se concreta también este artículo, pues:

Es en este campo donde a través de métodos sutiles de persuasión y disciplinamiento se educa a los cuerpos en materia de sexualidad y afectividad, y se configuran los habitus de socialización de género como "proceso mediante el cual el orden social se inscribe progresivamente en las mentes [...] y se encarna en nuestro cuerpo en forma de hexis corporal" (Rodríguez, 2003, citado en Silva-Segovia et al., 2015: 27).

El primer trabajo de referencia es el clásico de Willis (1988), un estudio etnográfico sobre la construcción de identidades masculinas adolescentes de clase obrera en el marco de los estudios culturales, mediante recursos simbólicos alternativos como ropa, música y ocio. El estudio inicial es actualizado a través de lo que Willis (2003) llama las tres grandes olas de modernización de la escuela, donde la máscara que es la masculinidad se va transformando para adaptarse al contexto socioestructural de cada ola. La primera se corresponde con las respuestas culturales a la escolarización universal en el contexto de la sociedad industrial, mediante una cultura de resistencia basada en una masculinidad ruda que confiere poder a los chicos sobre otros chicos menos exitosos y sobre las mujeres; una masculinidad compensatoria de la necesidad de sentirse superiores, donde trabajo manual se identifica con masculinidad. La segunda ola se caracteriza por las respuestas a la sociedad posindustrial, en 
un Reino Unido que comienza a perder trabajo industrial manual, disminuyendo la posibilidad de un salario decente para gran parte de la clase obrera y abriendo así una profunda crisis de la masculinidad obrera.

La tercera ola se corresponde con la cultura electrónica y de consumo (posmoderna), que llega hasta nuestros días. ${ }^{3}$ Se imponen valores como materialismo, dinero, sexo, violencia o comida rápida, que erosionan el valor y la dignidad humanos. Los significados producidos en la cultura de consumo interseccionan con otras formas de categorización social, produciendo posibilidades simultáneas de constricción y libertad que se expresan corporalmente a través de las raves, el hip-hop, la musculación, etc. La masculinidad resultante deja de estar asegurada por una herencia industrial proletaria, pero sigue dirigiéndose contra la escuela, derivando en misoginia y homofobia, criminalidad y violencia. Esa posición dominante es, sin embargo, preludio de su posterior subordinación en el mercado laboral.

Las masculinidades adolescentes de clase obrera en la Inglaterra posindustrial son analizadas también por Nayak (2006), quien señala igualmente la relación entre clase, género, raza y sexualidad, en una búsqueda continua de pertenencia, actualizando el trabajo de Willis (1988 y 2003) en la interpretación de los significados de la masculinidad de clase obrera, en la construcción de sentido cotidiano y de lo que llama "transiciones de juventud".

Por su parte, Richardson (2010) halla en su estudio que los jóvenes identifican "sexo" (heterosexual) con coito sobre todo. Define la sexualidad como un proceso social. Las prácticas sexuales adolescentes se enmarcan en relaciones y prácticas sociales más amplias que les confieren su significado, siendo la intimidad emocional el ámbito preferible para tener relaciones sexuales. La principal razón para tener sexo es ser aceptado por el grupo de iguales, habiendo una mayor presión sobre los chicos que sobre las chicas. Sin embargo, muchos jóvenes de su estudio reconocen que esperar a la chica correcta es la mejor opción; algunos incluso expresan su pesar por no haber esperado, aunque reconocen que "hacerlo" les causa alivio y orgullo.

De ahí la importancia de "hablar de sexualidad" como forma de interacción social que establece el marco donde se construyen las identidades (hetero)sexuales y de género, que se alcanzan colectivamente y producen jerarquías entre los chicos, convirtiendo experiencias sexuales privadas en actuaciones públicas. Se trata de los ritos de paso hacia la edad adulta que se negocian en el marco de la cultura de pares y que también Nayak (2006) observa en su trabajo.

3 Sin embargo, en el análisis de Willis (2003) no están aún las redes sociales ni la digitalización de la sociedad. 
En línea con Willis (1988 y 2003), Richardson (2010) retoma el análisis de las masculinidades y sexualidades de clase obrera para señalar que se construyen en relación, y a menudo en oposición, a otras formas de masculinidad, así como a las feminidades, por lo que tienen características específicas como el gusto por el deporte, peleas y atracción de las chicas, que derivan en una hipermasculinidad, pues no alcanzan las expectativas de las masculinidades de clase media que privilegian el éxito académico y el trabajo no manual.

Aunque Richardson (2010) no lo denomina como tal, en su análisis subyace también la construcción sexual de una masculinidad que es una máscara en cuya superficie prevalece la deseabilidad social en el grupo de amistad. De hecho, la literatura revisada muestra que las masculinidades heterosexuales dominantes se caracterizan por prácticas heterosexuales que son expresiones de homofobia y misoginia. Además, muchos jóvenes sienten que no pueden hablar de su falta de placer, ansiedades o preocupaciones sobre el sexo.

Esto nos remite, de nuevo, a la necesidad de observar "justo debajo de la superficie” (Haste, 2013), para comprender bien esa máscara que es la masculinidad, como "mensajes" portadores de la norma social de género (Harris, 1995). Como se observa en la mayoría de los estudios, las actitudes sexistas y homófobas son una estrategia para garantizar una "reputación heterosexual" exitosa entre los adolescentes (Forrest, 2000; Willis, 2003) frente a homosexuales y chicos considerados no masculinos (Haste, 2013). Ello implica también ser competente y experimentado en las relaciones sexuales coitales (Forrest, 2000; Richardson, 2010), sobre todo a partir de la percepción que se tiene de la norma a través de la familia y grupo de amistad (Barrera et al., 2004).

Por tanto, los estudios revisados muestran que la sexualidad masculina adolescente está definida por las marcas de género internalizadas mediante el proceso de socialización. En el presente estudio se analiza este fenómeno en el caso de España, donde apenas ha sido investigado, para poner el foco en la construcción de la masculinidad como máscara a través de los procesos de socialización y subjetivación sexual y de género, considerando también la pertenencia de clase.

\section{Objetivos y metodología de investigación}

Este artículo procede de tres investigaciones sobre las relaciones afectivosexuales de chicas y chicos de 12-18 años en la ciudad de Granada (Andalucía, España). Se trata de tres estudios de caso (Berg, 2001) realizados en 
2006, 2007 y 2014, en el ciclo de educación secundaria obligatoria. En todos se contó con el consentimiento informado de los centros educativos.

Los casos comprenden entrevistas, personales y en profundidad (Valles, 1997) a cada estudiante de los tres grupos clase (Venegas, 2011a), a fin de visibilizar las experiencias adolescentes a través de sus voces - un elemento central (Hadfield y Haw, 2001)-, para analizar la construcción de sus subjetividades (Silva-Segovia et al., 2015). Se ha adoptado, además, el enfoque de género (Heiskanen, 2006). Las entrevistas fueron grabadas y transcritas. En ellas se ha utilizado un protocolo de preguntas que comprende siete bloques relativos a las relaciones afectivosexuales de los sujetos participantes, así como a la educación afectivosexual recibida: familia, barrio, escuela, relaciones de pareja, relaciones afectivas, cuestiones de género y expectativas de futuro (Venegas, 2011b).

El objetivo general de este artículo es analizar las masculinidades adolescentes (12-18 años) y su representación de la sexualidad, entendida como "máscara" que se construye a través de los procesos de socialización y subjetivación de género (Venegas, 2017), para identificar las marcas de género que definen esos procesos. Por ello, el presente análisis se limita a las entrevistas con los chicos de los tres casos. En dos de estos (2006 y 2014), los jóvenes pertenecían a centros educativos situados en dos distritos considerados por el ayuntamiento de la ciudad de Granada como predominantemente de clase obrera, dado el perfil socioeconómico y educativo de su población, así como por el valor de la vivienda en sendos distritos o los niveles de (des) empleo. El tercer caso (2007), en pleno centro de la ciudad, es considerado por el ayuntamiento como predominantemente de clase media por esos mismos criterios. Se ha adoptado esta consideración municipal acerca de los distritos y, por ende, de los centros para situar la pertenencia de clase del entorno social del alumnado que asiste a cada uno de los tres centros educativos de secundaria.

Se analizaron las respuestas de un total de 26 chicos, cuyos perfiles sociodemográficos se muestran en la Tabla $1 ;{ }^{4}$ sus nombres son ficticios para garantizar el anonimato. El 69\% de los chicos entrevistados procede de entornos considerados de clase obrera, y $31 \%$ de clase media; $77 \%$ se define como caucásico, $15 \%$, gitano y $8 \%$, mestizo. En cuanto a la nacionalidad, $85 \%$ son españoles y sólo $15 \%$, extranjeros (Rumania, Rusia y Bolivia). Todos se declaran heterosexuales.

4 La tabla y los gráficos se encuentran en el Anexo, al final del presente artículo (Nota del editor). 
Dada la naturaleza de los datos analizados -cualitativa y localizada en tres casos-, el presente análisis no pretende llegar a conclusiones generalizables ni representativas de la totalidad de adolescentes españoles en la franja de edad estudiada, pero sí de tendencias sociales que pueden ser compartidas, generacionalmente, por los chicos en su misma situación social. Ahí radica el interés de este análisis en el marco del estudio de las relaciones afectivosexuales adolescentes desde una perspectiva feminista crítica, así como por su valor para una educación afectivosexual holística.

\section{Análisis de resultados: representación de la sexualidad y construcción de masculinidades adolescentes}

Los resultados fueron analizados desde la teoría del devenir sujeto entre la socialización y la subjetivación (Venegas, 2017), en el marco de un modelo holístico, democrático e igualitario de relaciones afectivosexuales adolescentes (Barragán, 1999; Sex Education Forum, 2006; Venegas, 2011c; Santos et al., 2012; Haste, 2013; Ketting e Ivanova, 2018). En este apartado se presentarán primero las dimensiones que representan la sexualidad en los discursos de los adolescentes entrevistados. A continuación se analizarán dichas dimensiones y se discutirán, entendiendo la masculinidad como una máscara normativa que han de habitar los chicos adolescentes, tal como se ha señalado en líneas anteriores.

Representación de la sexualidad y pertenencia de clase en las masculinidades adolescentes

El análisis de la sexualidad desde el enfoque de la clase social es un clásico en el estudio de las masculinidades, en especial de las adolescentes de clase obrera (Willis, 1988 y 2003; Nayak, 2006; Richardson, 2010; McCormack, 2014; Venegas, 2018). Los gráficos 1 y 2 muestran las dimensiones que representan la sexualidad para los chicos entrevistados, según la categorización de los datos de las entrevistas realizadas con ayuda del software Nudist NVivo. Esta categorización gráfica permite cuantificar la presencia de dichas dimensiones, en función de la clase social, lo cual nos posibilita observar algunas consideraciones de interés en perspectiva comparada.

Para todos los chicos, los ritos de paso aparecen como la dimensión más relevante en su representación de la sexualidad, si bien entre los adolescentes del colegio situado en el barrio de clase obrera su presencia es casi del doble 
que entre los del colegio de clase media (35\% y 19\%, respectivamente). Ello puede deberse al mayor número de embarazos adolescentes en el primer caso, así como a su entrada más temprana en el mercado laboral. También hay una diferencia considerable en torno al sentimiento de vergüenza ( $18 \%$ y $6 \%$, respectivamente), lo cual cabe atribuir a valores socioculturales aludidos en las entrevistas, como el honor y la honra. Finalmente, un aspecto referido sólo por los chicos del colegio ubicado en el barrio de clase obrera es el que hace mención expresa de la situación social del propio barrio, lo cual denota un fuerte sentimiento de pertenencia de clase:

Muchas veces, estás sentao y ves, no lo ves el vacío que te queda, lo ves como todo normal, muchos vienen del trabajo, otros pasan con carrillos llenos de chatarra y tó eso, las furgonetas llenas de cartones. No sé, a lo mejor, te vas pal [nombre de un centro comercial] y ves una manta y un colchón tó sucio, así, como de haber vivío ahí un vagamundo o lo que sea. Eso, al verlo, pos te impacta... Muchas veces te levantas pa ir al colegio y puedes ver, yo qué sé, a niños, a muchachos con 20, 23 años, tumbaos ahí en un colchón, tiraos, con cartones tapaos. Pos tú sales, lo primero que haces es fijarte. Y eso te impacta (Guille).

Algunas dimensiones aparecen sólo en las entrevistas con chicos del contexto de clase media, como el miedo al embarazo no deseado, seguramente porque en el contexto de clase obrera su mayor frecuencia lo vuelve un fenómeno socialmente "normalizado", así como la conciencia del papel que juega la socialización familiar en la sexualidad, o la referencia al grupo de amistad, que es relevante para los chicos del contexto de clase obrera en aspectos de la investigación que quedan fuera del objeto de este artículo. A continuación se analizan las dimensiones que representan la sexualidad de estos adolescentes en sus entrevistas y que dan contenido a los procesos de socialización y subjetivación en el devenir sujeto masculino adolescente.

Género y sexualidad, identidad y práctica, en la construcción de las masculinidades adolescentes

El género como norma social (Harris, 1995) estructura la representación de la sexualidad de los chicos entrevistados a través de su socialización de género. Siguiendo el esquema de Harris (1995), señalado antes, un primer aspecto que muestra cómo el género estructura dicha representación es la identificación de género a la hora de hablar de sexualidad en la familia. Los chicos prefieren claramente a sus padres: "Siempre con un hombre a lo mejor, como es de tu mismo sexo pues... y además tiene [su padre] cuarenta... [...] ya 
ha pasao por to lo que tú has pasao" (Fran $\left.{ }^{5}\right)$. Esa identificación es parte de una socialización diferencial de género desde el momento mismo de nacer, que los chicos entrevistados reconocen: "Siempre que nace alguien, o siempre que alguien está embarazao, compras o cosa azul o cosa rosa” (Guille). Ahí radica el origen de la desigualdad de género, que activa la construcción de identidad y prácticas generizadas, según la norma social de género. Se construye así una masculinidad dominante que, en las entrevistas, se materializa en un doble estándar de género en dos aspectos:

1. El ejercicio del poder (Connell, 1987; Harris, 1995; Willis, 1988 y 2003):

Yo creo que deben respetar de una manera a los hombres y de otra... [...] es por la forma de mi familia, porque siempre mi madre es "que voy a hacer esto" [...] de más chico, que decía "voy a venir a las dos de la mañana". Pos "pregúntale a tú padre", pos veo a mi padre, que lo que él dice es lo que se hace. Si yo a mi madre le digo una cosa y me dice "venga pos sí, o pos no"... Pero es que tó es "a tu padre" (Javi).

2. La práctica de la sexualidad (Forrest, 2000; Barrera et al., 2004; Richardson, 2010):

Si fuera niña, no sería igual, porque si yo soy padre y tengo un niño, y mi niño se quiere ir tirando a toas, digo que me molestaría porque es mi niño, así, en general, pero un niño, como que no va a venir embarazao. Pero si fuera una niña, yo no quiero que mi niña se haga la puta del pueblo. Porque es diferente, es por la sociedad, no porque yo sea machista, sino que la sociedad lo ve así (Tony).

La máxima expresión de esa generización de las prácticas afectivosexuales es, según revelan las entrevistas, la violencia de género:

Esa niña va a terminar hecha... loca de la cabeza. El novio la trata como una mierda. [...] Tó el mundo se lo dice: "tú estás gilipollas de la cabeza". Hasta las amigas y de tó. [...] La trata como una mierda. [...] Esa niña va a acabar hecha pedazos. Se la van a cargar (Luis).

El resultado es la construcción de una máscara -la masculinidad-, la cual, al tiempo que confiere el poder ya señalado, constriñe a los chicos, impidiendo que puedan reconocer sus dudas y falta de conocimiento, por temor a que su virilidad sea cuestionada (Sex Education Forum, 2006; Richardson, 2010; Haste, 2013):

Mucha gente tiene dudas y no se va al hospital a preguntarlo. [...] a lo mejor, alguno no sabe ponerse un preservativo y por tal de no ir a preguntar a fulanico, o no ir a preguntarle, correctamente, se callan y se quedan con la duda. Y eso pasa porque vamos... a lo mejor hay algunos que van así "no sé qué", pero luego le preguntas "y oye, tú, no sé qué", y no es por hacerte tú el listo, es pa ver si lo sabe de verdad y a lo mejor dice: "sí, pues ha sío así", y dices "no" (Fran).

5 Los verbatims recogen la transcripción literal de la expresión de los sujetos entrevistados. 
El habla misma, como práctica que vehicula la identidad y permite la comunicación, es reflejo de esa máscara generizada que opta por términos y expresiones rudas (Willis, 1988; Harris, 1995; Forrest, 2000) como muestra de virilidad (Bourdieu, 2005):

Si tó la gente van a su bola, van a su rollo, por ejemplo, te dicen algo "tonto, no sé qué, no sé cuánto", "cállate, que la próxima vez voy", "has oído a éste", "bah, que le den por culo"... Y así (Mario).

Esta máscara internalizada a través de la socialización orienta prácticas de acomodación a la norma de género entre los chicos entrevistados, pero también de resistencia, activando un proceso de subjetivación que define el "devenir sujeto" (Venegas, 2017) masculino en el conflicto entre la adhesión a esa norma y la ruptura con la misma, en la búsqueda por negociar los términos en que van construyendo su propia masculinidad, en "transición" hacia la edad adulta (Nayak, 2006). De ahí que sean a la vez beneficiados por el patriarcado y críticos con el sexismo que genera:

Yo sé que hay muchísimos en la clase que son muy machistas, pero muy machistas. Y yo he llegao a oír barbaridades como "las niñas que tienen novio, no tienen por qué salir a la calle”, y quedarse tó la clase pará... [...] y las niñas “ipero bueno!", gritando "ah, pos no salgas tú, no sé qué" (Alberto).

La iniciación en la práctica del coito - el mito de la primera vez- es la práctica sexual que ocupa el espacio central en las entrevistas al "hablar de sexualidad” (Richardson, 2010). La edad media de la primera relación coital en Europa es a los 15 años (Ketting e Ivanova, 2018). Se trata de una práctica influida por la presión en el grupo de amistad (Barrera et al., 2004; Richardson, 2010) a edades tempranas (Baumer y South, 2001, citado en Barrera et al., 2004). Sin embargo, las entrevistas revelan el malestar derivado de esta presión sobre los chicos (Forrest, 2000; Haste, 2013). La masculinidad dominante, experta en sexualidad y siempre dispuesta a la práctica sexual, es una máscara que subyace "justo debajo de la superficie” (Blake, 2004: 281, citado en Haste, 2013: 523) del liderazgo y el placer sexuales, derivados de los "mensajes que escuchan los hombres" (Harris, 1995) sobre sexualidad:

Lo intentamos hacer una vez mi novia y no lo conseguimos. [...] Un niño dice: "vamos a hacerlo", "pos vamos", está siempre preparáo. [...] A lo mejor a una niña se lo dices y yo sé que... Yo se lo he dicho muchas veces: "yo sé que te lo tienes que pensar, yo no te meto prisa”. [...] Y una vez me dejó por eso. [...] Hubo otro día que intenté con mi novia, bueno, no lo intenté, pasó así... y no tenía preservativo ni ná, y dije que no, y cogí, y estaba a puntito, porque la otra vez lo intentamos y no entró [...]. Porque me lo dijo ella [...] estamos tumbaos y me dice: "es que te tengo que decir una cosa". Me vuelvo, y yo "tú verás lo que me va a decir". Y dice: "quiero que sea contigo. ¿Me quieres?”. Me 
quedo así, y al principio no lo pillé y, de pronto, no lo pillo y dice: "que sí, que quiero que sea contigo". Y enseguida me vino un flash así, y dije "ostias, no sé qué". Muchas veces había soñao con eso, pero nunca creí [...] que me lo iba a decir ese día. Y ya cogió y me dice: "que quiero que sea contigo". Y me quedo así... Y yo: “¿tú estás segura?”. Y me dice: "sí”. Y digo: "¿estás segura?", "que sí, que sí". Y lo intentamos, y no pasó ná. Luego, justamente el día que no lo intentamos, que la tenía casi dentro, ¿̇abes? [...] le dije que no. Bueno, no le dije que no, la retiré [...] Hice así... y dije: "no, que no, espera". Así que fue sin ná, y siendo la primera vez para ella, tampoco... [...] Que yo tampoco estoy mu convencío de hacerlo con ella... (Fran).

Deseo sexual y amor romántico en la adolescencia se (con)funden, construyendo el mito de la primera vez, que genera dudas entre los chicos entrevistados. La cita anterior ejemplifica el conflicto entre normas internalizadas en la socialización sexual generizada y los valores de resistencia a ese modelo de masculinidad dominante que activa el devenir sujeto agente, sujeto de la acción, a través de una subjetivación (Venegas, 2017) por la cual el chico se va posicionando, tomando decisiones y acercándose a un modelo de masculinidad propio, donde identidad y prácticas se complementan en la representación y la vivencia de la sexualidad que conduce a la edad adulta, como se analiza en el siguiente apartado.

\section{La sexualidad como transición: los ritos de paso}

La sexualidad marca varios ritos de paso, especialmente en la adolescencia (Richardson, 2010), que describen un proceso de construcción y búsqueda de pertenencia en línea con lo que Nayak (2006) denomina "transiciones de juventud".

Primero, la transición de la infancia a la adolescencia, mediante la pubertad y el despertar del deseo sexual, que se mezcla y se complica con el enamoramiento en las relaciones afectivosexuales adolescentes:

A mí antes me gustaban mucho las Plays. Yo recuerdo que antes me iba con mis amigos a jugar al fútbol. De repente, te echas novia, y ya no piensas en la Play, piensas en la novia [...]. Empiezas a sentir sentimientos de estos más chungos, que tú no has sentío [...] porque si estás enamorao, es una cosa diferente a no estarlo [...] Cuando vas siendo más grande, necesitas más dinero, te vas dando cuenta de que todo el día con la Play no puedes estar, o jugando al fútbol, ya tienes que buscarte la vida, ganarte ese dinero, comprarte ropa, a lo mejor una casa, trabajar... (Rubén).

El segundo rito de paso, contenido ya en el momento anterior, supone la transición definitiva de la adolescencia a la edad adulta, la estabilización de una pareja, la paternidad y la asunción de responsabilidades: 
Yo hasta que no tenga canas no quiero tener ningún niño. [...] Yo voy a tener un niño cuando sepa que voy a estar con él tol día. [...] cuando le pueda dar un trozo de pan y cuando pueda darle un techo y cuando yo tenga tos mis cosas estables, y siempre que queramos, no sé, yo creo que en un tiempo sí podría ser buen padre, pero es que no lo sé tampoco, son muchos los que fracasan en el intento (Luis).

La paternidad sustituye al deseo sexual como hito de esas transiciones y culmina en la masculinidad adulta. ${ }^{6}$ Relacionado con ello, cabe mencionar el embarazo adolescente, un temor destacado para los chicos de clase media, como muestra el anterior verbatim, de Luis, pero rito de paso para los chicos de clase obrera:

No se han casao, es que digo su marío porque cuando se quea preñá, es su marío [...]. Parece que hay un virus o algo, que se han quedao tó las jóvenes preñás [...]. Tienen toas un año o dos más que yo [...] Lo ven más raro que lleguen vírgenes a casarse, que llegar preñá (Raúl).

En todo caso, es siempre objeto de negociación de poder en la pareja adolescente, como muestran varios estudios (Holland et al., 1990; Santos et al., 2012), conforme a los cuales no siempre se toman medidas anticonceptivas adecuadas, aun cuando se conocen. El rechazo, sobre todo al preservativo, se debe a que rompe la espontaneidad y fluidez del coito, según señalan los sujetos:

Yo tengo un puñao de miedo a que mi novia se quede embarazá. Yo tengo mucho miedo por eso y tó las veces estoy rallao y mi novia pos no es miedosa ni ná, como otras novias. [...] Antes sí, lo utilizábamos [preservativo]. Pero ya no [...] Si eso ya lo sé yo todo [sobre anticonceptivos], pero es que, yo qué sé, ya es que... porque no voy dentro y yo creo que lo voy a pasar bien... pero que estoy mu cagao siempre (Tony).

\section{Vergüenza y dignidad: la sexualidad como algo sucio}

Como muestran los resultados, la sexualidad adolescente está definida por normas que generan continuos conflictos en estos chicos entre el deseo y el tabú, la libertad y la constricción. Ejemplo de ello es el sentimiento de vergüenza que vertebra varios aspectos referidos en las entrevistas. Para algunos de los chicos, esta vergüenza es fruto de identificar sexualidad con tabú, lo cual define la socialización sexual que construye sus masculinidades según una mayor, menor o nula resistencia a las normas de esa socialización:

6 El estudio del cambio de modelos de paternidad (Barbeta-Viñas y Cano, 2017) sería un ejemplo del estudio más amplio de las masculinidades. La paternidad ejemplifica y concreta la masculinidad. La transición en la paternidad hacia un modelo de crianza más igualitario significa un cambio hacia una masculinidad más igualitaria. 
A lo mejor estamos viendo en la tele un programa que habla de acto sexual. Pos tú estás dando unas cuantas cosas aquí, ¿no ?7 Lo decía y se pone mi padre: "niño, eso no se dice, eso se guarda pa ti, no sé qué”. Se pone mi madre: “pos si lo sabe, qué más da que lo diga, eso es bueno, que se sepan esas cosas, pa cuando llegue en un futuro, pos que sepa lo que tiene que hacer". [...] Mi padre se ponía por las nubes. Se ponía a dar voces: "niño, que eres un guarro, no sé qué. Que esas cosas son pa ti, no lo digas”. Me echó una que no veas (Guillermo).

De ahí una segunda dimensión de la vergüenza: optar por el uso de eufemismos en lugar de denominar a los diversos aspectos de la sexualidad por su nombre: "Me puso la mano en las partes prohibidas de las niñas [...] Yo digo 'si me ha dejao que le toque esto, me dejará que le toque otras cosas"” (Raúl).

Finalmente, los resultados revelan un tipo de vergüenza connotado por el valor de la honra como ideal para determinados grupos étnicos, por ejemplo la población gitana: "No la quiero, a lo mejor, la dignidad se la he quitao yo y yo no le correspondo, ¿ tú me entiendes? Por eso me siento sucio” (Raúl). Todas estas dimensiones de la vergüenza ejercen una función de control social (Harre y Parrott, 1996, citado en Forrest, 2000), a través de la sexualidad como institución de regulación social (Weeks, 2003), que es central en la construcción de identidades y prácticas masculinas en la adolescencia.

\section{El grupo de amistad}

El grupo de amistad es la agencia social más significativa en la adolescencia, esencial para el descubrimiento, la vivencia y la experiencia de la sexualidad (Forrest, 2000; Barrera et al., 2004; Richardson, 2010; Haste, 2013; Venegas, 2018). En las entrevistadas analizadas es aludida por los chicos de clase media pero no por los de clase obrera, que sí lo hacen en referencia a otros aspectos no abordados en este artículo. Una experiencia destacada es el descubrimiento de la sexualidad mediante las primeras prácticas sexuales en grupo:

A descubrir la sexualidad, o sea, lo que es empezar yo a masturbarme y eso, pos no me acuerdo, sería a los doce, o a los once. Me parece que fui a casa de mi vecino y me dijo: "te voy a enseñar una cosa", y me puso un vídeo porno y me dice: "venga, vamos a hacernos una paja”. Y yo: "¿una paja? ¿Eso qué es?”. No sabía lo que era una paja. "Joé, eso es hacer así, así". Porque mi vecino tampoco sabía y digo: "ah". Nos pusimos allí los dos tonticos a hacer así, así, y ya pues... (Alberto).

7 Se refiere al curso de educación afectivosexual realizado con cada uno de los tres grupos de los tres estudios, previo a las entrevistas personales en profundidad. 
"Hablar de sexualidad" con los amigos se convierte en una práctica central en el grupo de amistad (también en Haste, 2013), ocupando buena parte de sus conversaciones frente a la dificultad que encuentran para ello en otras agencias sociales, donde es casi tabú, según la percepción de los chicos entrevistados:

Es un tema ahora de conversación entre los chavales, que si "ah, te metes en esta página porno, que tal y que cual". Pues me tengo que meter más en el ambiente, pero que en mi casa se habla bastante poco de lo que es el sexo, no es que sea un tabú, pero se habla bastante poco (Antonio).

La edad juega un papel fundamental. La veteranía es sinónimo de experiencia, confiere estatus y determina la posición en la jerarquía de poder del grupo de amistad (Richardson, 2010). El grupo "educa” para la sexualidad:

Allí es que tenemos tós mucha confianza, es que somos tós un grupo. Nosotros, los chiquitillos, nos juntamos con los grandes, los grandes con los más grandes. [...] Desde los 10 hasta el más viejo que puede tener 60 . El que lo sabe es el que habla más, y los demás escuchamos (Rubén).

Hablar de sexualidad con los amigos es también motivo de diversión y objeto de bromas, pero los chicos conocen las normas sociales sobre sexualidad, por lo cual cuestionan las prácticas que rebasan los límites de esas normas:

Hay algunos que se desfasan mucho ya. [...] Empiezan a hablar y empiezan a decirte que se han ido de putas, que eso no lo van a volver a hacer... [...] Y de meterse los dos en la misma habitación... Yo lo he visto en el vídeo, grabao. [...] Son los tíos más follarines del mundo, ¿sabes lo que te digo? Son niños que te cuentan cosas que han hecho con esa edad y dices: " $\mathrm{iMe}$ cago en la puta, pero vosotros pa qué vais, vais pa depravados mentales". [...] Cuando pillan dineros, pillan y se van de putas. [...] Pillan a las niñas y hacen tríos con ellas y de tó, y las niñas se dejan, y lo cuentan y es que te partes (Luis).

Estos discursos revelan una socialización en un modelo de masculinidad hegemónica, donde la práctica sexual, principalmente la coital, y también el consumo de pornografía y prostitución se sitúan en el centro de la construcción de su ser hombres heterosexuales, sexualmente activos. Esto les confiere su posición en la jerarquía de poder en el grupo de amistad, al tiempo que se revela una subjetivación por la cual los adolescentes se resisten igualmente a esa masculinidad hegemónica, llegando a cuestionar las prácticas sexuales que exceden los límites de la norma sexual generizada.

\section{Misoginia y homofobia}

Como señalan los estudios revisados (Willis, 1988 y 2003; Forrest, 2000), misoginia y homofobia son dos lugares comunes en las masculinidades 
hegemónicas en la adolescencia. Un ejemplo frecuente de misoginia reside en el doble estándar con que contemplan las prácticas sexuales de las chicas que disfrutan de su sexualidad, como muchos de ellos hacen:

Algunas niñas que son un poco putas, que a lo mejor llegan un día y se leían con uno, y al día siguiente con otro, y al día siguiente con otro (Sergio).

La diversidad sexual constituye la mayor controversia en las entrevistas realizadas, donde hay abundantes ejemplos de discriminación sutil:

Mucha gente aquí en el colegio que dice: "no, no, con los gays bien", y el primer insulto que se le viene a la boca es maricón. Y dices: "bueno, ya estás teniendo un prejuicio al decir eso. Si tanto hablas, de que tú...”. Y gente que, bueno, que ya es que ni escucharlos, que te dice: "yo es que no podría tener un amigo gay". Y dices "pero, ¿por qué?". "No, vayamos a que me meta mano". Y es que parece como si tos los gays fueran unos salíos. Y son personas como nosotros (Alberto).

Un análisis más detallado de las entrevistas muestra, sin embargo, un rechazo más explícito a identidades y prácticas no heterosexuales, que parecen ser relativamente aceptadas cuando se producen en el ámbito privado de los sujetos, pero no si se hace alarde de ello en público:

En mi banda, pues sí, hay un gay, y sí lo tragan, pero yo qué sé. Es que no es gay, es maricona loca, que es de las que se creen mujer, no es que se crea mujer, es que es una cosa mu rara, como si tú eres gay, y tú eres gay y dices que eres gay, ¡coño!, te respetarán. Si vas dando voces y con las niñas y tó, digo: “idónde va esto?” (Tony).

Las entrevistas ponen de manifiesto que el origen de esa homofobia es, a menudo, la propia socialización en el ámbito familiar:

Mi abuelo no soporta a los mariquitas. Mi abuelo es cerrao. Dice que después de tener un niño drogao, tener un niño maricón es... eso. Mi tío tampoco los soporta. Como es mu, mu, mu macho, le da asco. Si yo, por ejemplo, le hago bromas y de tó: “Ay, qué culillo más bonico tiene!". Y lo que es bromas de mariquitas lo soporta, pero... [...]. Me dan asco los hombres, no es que me den asco [pensando rápido], sí, me dan asco, pero no es eso, me dan, esos pelos en el culo, por Dios y por la virgen, iqué asco más grande! (Raúl).

La pertenencia étnica parece ser uno de los factores explicativos de este rechazo a la diversidad sexual y las formas de vivirla. Frente a ello, los chicos entrevistados reconocen también la importancia de la educación afectivosexual como una de las mejores herramientas para combatir la falta de respeto a las personas por razón de su identidad sexual y/o de género, y su orientación sexual, aunque todavía hay trabajo educativo por hacer:

Porque yo antes a los gays, los veía que... no podía ni verlos. Pero viendo [...] lo que tú has dicho, pues... Si ellos son así, pos que sean así, pero que yo no quiero saber ná de ellos, porque yo tengo un amigo mío [...] tiene novio y tó, y es el niño más buena gente que existe, ese niño es buenísima gente y tiene su novio, pero es distinto, tiene su novio, pero no... (Javi). 
También Forrest (2000) encuentra mayor hostilidad entre los chicos que entre las chicas para trabajar los prejuicios en torno a la homosexualidad. Sin embargo, estudios como el de McCormack (2014) ponen de manifiesto una disminución de las actitudes homófobas por parte de los chicos de clase obrera, lo cual podría atribuirse a una apuesta por la educación afectivosexual donde el reconocimiento a la diversidad sexual ocupa un espacio central (Sex Education Forum, 2006; Ketting e Ivanova, 2018).

\section{Discusión y conclusiones}

En la actualidad, los estudios feministas y de género sitúan el análisis de las masculinidades en el centro de los debates sobre democracia, igualdad y justicia social. En la misma línea, el objetivo de este artículo ha sido analizar la representación de la sexualidad en las masculinidades adolescentes, atendiendo a su contexto social de referencia (clase social). Para ello, se ha seguido la teoría del devenir sujeto masculino adolescente a través de los procesos complementarios de socialización y subjetivación sexual y de género que, a su vez, están relacionados con procesos de reproducción y cambio social (Venegas, 2017). De ahí la necesidad de examinar esos procesos del devenir sujeto que subyacen bajo la superficie de la masculinidad definida aquí como máscara.

Para ello, el artículo ha comenzado analizando los modelos patriarcales de masculinidad hegemónica (Connell, 1987) o dominante (Bourdieu, 2005), según las normas sociales de género (Harris, 1995) que, junto al privilegio general concedido a los hombres, imponen una jerarquía interna a esta categoría social. Más recientemente se ha empezado a hablar de masculinidad inclusiva (Anderson y McCormack, 2018).

Con este marco de fondo, el artículo ha ofrecido un análisis basado en 26 entrevistas personales en profundidad procedentes de tres estudios de caso, para conocer la máscara que son las masculinidades adolescentes (1218 años). Los resultados revelan que la sexualidad es para los adolescentes un espacio social donde construyen su masculinidad como una máscara erigida sobre el conflicto entre el deseo sexual, la presión por ser sexualmente activo, las prácticas sexuales -especialmente coitales- y los temores, miedos, dudas y angustias que todo ello suscita, aderezado además por sentimientos de enamoramiento y amor romántico, en el contexto de sus relaciones afectivosexuales.

Las dimensiones que representan la sexualidad en los discursos de los adolescentes entrevistados dan cuenta de los mensajes -las normas sociales 
de género- que escuchan los chicos (Harris, 1995) desde que nacen, y que dirigen el proceso del devenir sujeto (Venegas, 2017). Así se activa la construcción de su masculinidad como una máscara (Haste, 2013) en la que está presente también la pertenencia de clase.

$\mathrm{Al}$ analizar qué subyace bajo la superficie de esa máscara, hallamos que no se trata de un proceso plano y lineal, sino la activación de procesos de adhesión, acomodación y ruptura con la norma de género, materializada por la masculinidad dominante, en la búsqueda hacia su formación como "hombre real" (Sex Education Forum, 2006) en la edad adulta. En estos procesos, clase, género y etnia interseccionan como los tres principios estructurantes de la identidad y las prácticas sexuales adolescentes más relevantes (Venegas, 2018).

La máscara que es la masculinidad adolescente es el resultado del conflicto y las contradicciones con que los sujetos adolescentes gestionan, por un lado, las normas internalizadas en su socialización sexual y de género, y, por otro, las que dan contenido a la resistencia que se activa en su subjetivación sexual y de género. La masculinidad adolescente es así un modelo de ser sujeto social masculino, de devenir hombre, en el cual -en el caso concreto en este artículo- la sexualidad patriarcal hegemónica, sexista, homófoba, ruda, sin erosiones ni grietas, sin dudas, sin mostrar emociones ni debilidad (Willis, 1988 y 2003; Connell y Messerschmidt, 2005; Bourdieu, 2005; Forrest, 2000; Richardson, 2010), internalizada en la socialización, choca cada vez más con una masculinidad inclusiva, (Anderson y McCormack, 2018) que da muestras de superar de forma paulatina esos valores limitantes, desigualitarios, como resultado de una subjetivación nutrida precisamente de los valores que ofrece un modelo alternativo de masculinidad.

Para tal fin -y con esa reivindicación se concluye este artículo- es imprescindible apostar por un modelo comprensivo de educación afectivosexual (Ketting e Ivanova, 2018), holístico, democrático, que promueva la igualdad sexual y de género, y con ello la justicia social (Venegas, 2011c). 


\section{Referencias}

Anderson, Eric y McCormack, Mark (2018), "Inclusive Masculinity Theory: overview, reflection and refinement", en Journal of Gender Studies, núm. 5, Reino Unido: Routledge.

Barbeta-Viñas, Marc y Cano, Tomás (2017), “¿Hacia un nuevo modelo de paternidad? Discursos sobre el proceso de implicación paterna en la España urbana”, en Revista Española de Investigaciones Sociológicas, España: Centro de Investigaciones Sociológicas.

Barragán, Fernando (1999), Programa de Educación Afectivo Sexual. Educación Secundaria, España: Consejería de Educación y Ciencia e Instituto Andaluz de la Mujer.

Barrera, Fernando et al. (2004), "Relación de las actitudes personales y de la norma social con la actividad sexual de los adolescentes", en Revista de Estudios Sociales, núm. 7 , Colombia: Universidad de los Andes.

Berg, Bruce (2001), Qualitative Research Methodsfor the Social Sciences, Reino Unido: Allyn $\&$ Bacon.

Bourdieu, Pierre (2005), La masculinidad hegemónica, España: Anagrama.

Butler, Judith (1997), The psychic life of power. Theories in subjection, Estados Unidos: Stanford University Press.

Connell, Robert (1987), Gender and Power. Society, the Person and Sexual Politics, Estados Unidos: Stanford University Press.

Connell, Robert (1997), “Gender politics for men”, en The International Journal of Sociology and Social Policy, núm. 1-2, Reino Unido: Emerald Publishing Limited.

Connell, Robert y Messerschmidt, James (2005), "Hegemonic Masculinity: Rethinking the Concept”, en Gender and Society, núm. 6, Estados Unidos: Sage.

Crenshaw, Kimberle (1989), "Demarginalizing the Intersection of Race and Sex: A Black Feminist Critique of Antidiscrimination Doctrine, Feminist Theory and Antiracist Politics", en University of Chicago Legal Forum, núm. 8, Estados Unidos: University of Chicago.

Esteban, Mari Luz (2001), “El género como categoría analítica. Revisiones y aplicaciones a la salud”, en Miqueo, Consuelo et al. [comps.], Perspectivas de género en salud. Fundamentos cientificos y socio-profesionales de diferencias sexuales no previstas, España: Minerva Ediciones.

Forrest, Simon (2000), "Big and tough: Boys learning about sexuality and manhood", en Sexual and Relationship Therapy, núm. 3, Reino Unido: Routledge.

Gómez, Águeda et al. (2016), "Dominación, sexualidad masculina y prostitución en España: ¿por qué los hombres españoles consumen sexo de pago?”, en Convergencia. Revista de Ciencias Sociales, núm. 71, México: Universidad Autónoma del Estado de México.

Hadfield, Mark y Haw, Kaye (2001), "Voice', young people and action research", en Educational Action Research, núm. 3, Reino Unido: Routledge.

Harding, Sandra (1986), The Science Question in Feminism, Estados Unidos: Cornell University Press.

Harris, Ian (1995), Messages men hear. Constructing masculinities, Reino Unido: Taylor \& Francis.

Haste, Polly (2013), "Sex education and masculinity: the 'problem' of boys", en Gender and Education, núm. 4, Reino Unido: Routledge. 
Convergencia Revista de Ciencias Sociales, vol. 27, 2020, Universidad Autónoma del Estado de México

Haywood, Chris y Mac an Ghaill, Máirtín (2012), “'What's next for masculinity?' Reflexive directions for theory and research on masculinity and education”, en Gender and Education, núm. 6, Reino Unido: Routledge.

Heiskanen, Tuula (2006), "Gender Issues in Action Research: Implications for Adult Education”, en International Journal of Lifelong Education, núm. 5, Reino Unido: Routledge.

Holland, Janet et al. (1990), “Don't die of ignorance' I nearly died of embarrassment. condoms in context”, en Wrappaper, núm. 2, Reino Unido: Tufnell Press.

Ketting, Evert e Ivanova, Olena (2018), Sexuality Education in Europe and Central Asia. State of the Art and Recent Developments. An Overview of 25 Countries, Alemania: Federal Centre for Health Education e International Planned Parenthood Federation European Network.

Maquieira, Virginia (2001), "Género, diferencia y desigualdad", en Beltrán, Cristina y Maquieira, Virgina [comps.], Feminismos, España: Alianza.

McCormack, Mark (2014), "The intersection of youth masculinities, decreasing homophobia and class: an ethnography", en The British Journal of Sociology, núm. 1, Reino Unido: Wiley-Blackwell Publishing.

Moore, Henrietta (1999), Antropología y feminismo, España: Cátedra Feminismos.

Narotzky, Susana (1995), Mujer, mujeres, género. Una aproximación critica al estudio de las mujeres en las ciencias sociales, España: Consejo Superior de Investigaciones Científicas.

Nayak, Anoop (2006), "Displaced Masculinities: Chavs, Youth and Class in the Postindustrial City”, en Sociology, núm. 5, Reino Unido: Sage.

Richardson, Diane (2010), "Youth masculinities: compelling male heterosexuality", en The British Journal of Sociology, núm. 4, Reino Unido: Wiley-Blackwell Publishing.

Rubin, Gayle (1975), “The Traffic in Women: Notes on the 'Political Economy' of Sex”, en Reiter, Rayna [comp.], Toward and Anthropology of Women, Reino Unido: Monthly Review Press.

Santos, Sofia et al. (2012), "Sex Education and the Views of Young People on Gender and Sexuality in Portuguese Schools", en Educação, Sociedade \& Culturas, Portugal: Universidade do Porto.

Silva-Segovia, Jimena et al. (2015), "Discursos sobre normas relativas a sexualidad en jóvenes del norte de Chile”, en Convergencia. Revista de Ciencias Sociales, núm. 67, México: Universidad Autónoma del Estado de México.

Sex Education Forum (2006), "Boys and young men: Developing effective sex and relationships education in schools", en Updating Forum Factsheet 11, Reino Unido: National Children's Bureau.

Touraine, Alain (2005), Un nuevo paradigma para comprender el mundo de hoy, España: Paidós.

Thurén, Britt-Marie (1993), El poder generizado. El desarrollo de la antropología feminista, España: Universidad Complutense.

Valles, Miguel (1997), Técnicas cualitativas de investigación social. Reflexión metodológica y práctica profesional, España: Síntesis.

Venegas, Mar (2011a), "La investigación acción educativa en educación afectivosexual: una metodología para el cambio social”, en EMPIRIA. Revista de Metodología de las Ciencias Sociales, núm. 1, España: Universidad Nacional de Educación a Distancia. 
Venegas, Mar (2011b), "Un modelo sociológico para investigar las relaciones afectivosexuales”, en Revista Mexicana de Sociología, núm. 4, México: Universidad Nacional Autónoma de México.

Venegas, Mar (2011c), "El modelo actual de educación afectivosexual en España. El caso de Andalucía”, en Revista Ibero-americana de Educação, núm. 3, España: Organización de Estados Iberoamericanos.

Venegas, Mar (2017), “Devenir sujeto. Una aproximación sociológica”, en Convergencia. Revista de Ciencias Sociales, núm. 73, México: Universidad Autónoma del Estado de México.

Venegas, Mar (2018), "El romance adolescente. Un análisis sociológico de la política afectivosexual en la adolescencia”, en Papers. Revista de Sociología, núm. 2, España: Universidad Autónoma de Barcelona.

Verloo, Mieke (2006), "Multiple Inequalities, Intersectionality and the European Union”, en European Journal of Women's Studies, núm. 3, Reino Unido: Sage.

Weeks, Jeffrey (2003), Sexuality, Reino Unido: Routledge.

Willis, Paul (1988), Aprendiendo a Trabajar, España: Akal.

Willis, Paul (2003), "Foot Soldiers of Modernity: The Dialectics of Cultural Consumption and the 21st-Century School", en Harvard Educational Review, núm. 3, Estados Unidos: Harvard University. 
Anexo

\section{Tabla I}

Perfil de los sujetos entrevistados en las tres investigaciones

\begin{tabular}{|c|c|c|c|c|c|}
\hline Nombre ficticio & Edad & Clase social & Etnia & Año & Nacionalidad \\
\hline Mario & 15 & Obrera & Caucásica & 2006 & Española \\
\hline Raúl & 17 & Obrera & Mestiza & 2006 & Española \\
\hline Raimundo & 18 & Obrera & Gitana & 2006 & Española \\
\hline Guille & 17 & Obrera & Caucásica & 2006 & Española \\
\hline Jose & 15 & Obrera & Caucásica & 2006 & Española \\
\hline Jorge & 16 & Media & Caucásica & 2006 & Española \\
\hline Elías & 17 & Obrera & Caucásica & 2006 & Española \\
\hline Fran & 17 & Media & Caucásica & 2007 & Española \\
\hline Alberto & 15 & Media & Caucásica & 2007 & Española \\
\hline Juan & 16 & Media & Caucásica & 2007 & Española \\
\hline Antonio & 15 & Media & Caucásica & 2007 & Española \\
\hline Sergio & 15 & Media & Caucásica & 2007 & Española \\
\hline Luis & 17 & Media & Caucásica & 2007 & Española \\
\hline Tony & 17 & Media & Mestiza & 2007 & Española \\
\hline Javi & 17 & Obrera & Caucásica & 2007 & Española \\
\hline Abel & 14 & Obrera & Caucásica & 2014 & Rumana \\
\hline Ernesto & 13 & Obrera & NS/NC & 2014 & Boliviana \\
\hline Jesús & 12 & Obrera & Gitana & 2014 & Española \\
\hline Pablo & 12 & Obrera & Caucásica & 2014 & Rusa \\
\hline Julio & 15 & Obrera & Gitana & 2014 & Española \\
\hline Salva & 14 & Obrera & Caucásica & 2014 & Española \\
\hline César & 14 & Obrera & Caucásica & 2014 & Española \\
\hline Fidel & 16 & Obrera & Caucásica & 2014 & Rumana \\
\hline Nacho & 14 & Obrera & Caucásica & 2014 & Española \\
\hline Andrés & 17 & Obrera & Caucásica & 2014 & Española \\
\hline Rubén & 16 & Obrera & Gitana & 2014 & Española \\
\hline
\end{tabular}

Fuente: Elaboración propia a partir de las entrevistas realizadas. 
Mar Venegas. La masculinidad como máscara: clase, género y sexualidad en las masculinidades adolescentes

\section{Gráfico 1}

Dimensiones que representan la sexualidad en los discursos de los sujetos de clase media

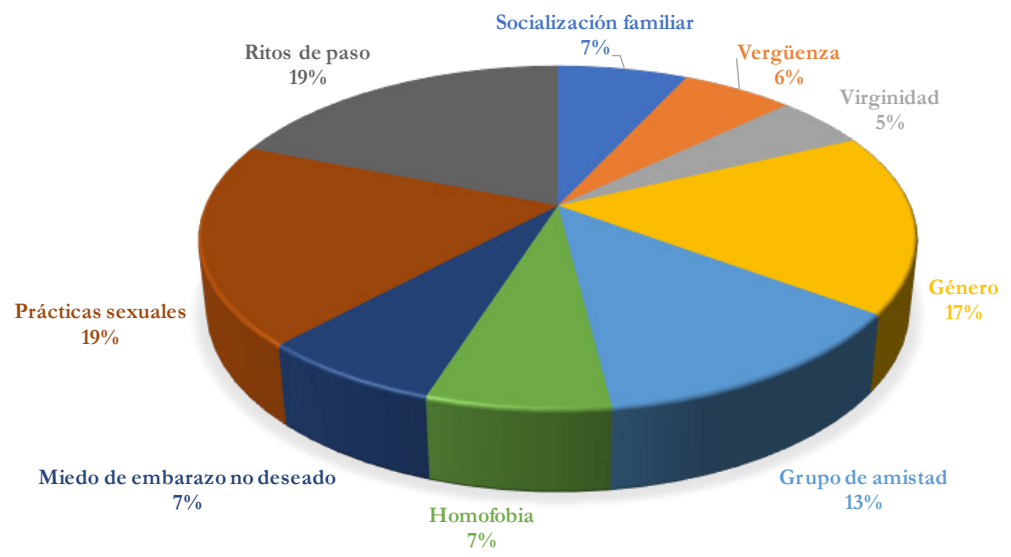

Fuente: Elaboración propia a partir de las entrevistas realizadas. 


\section{Gráfico 2}

Dimensiones que representan la sexualidad en los discursos de los sujetos de clase obrera

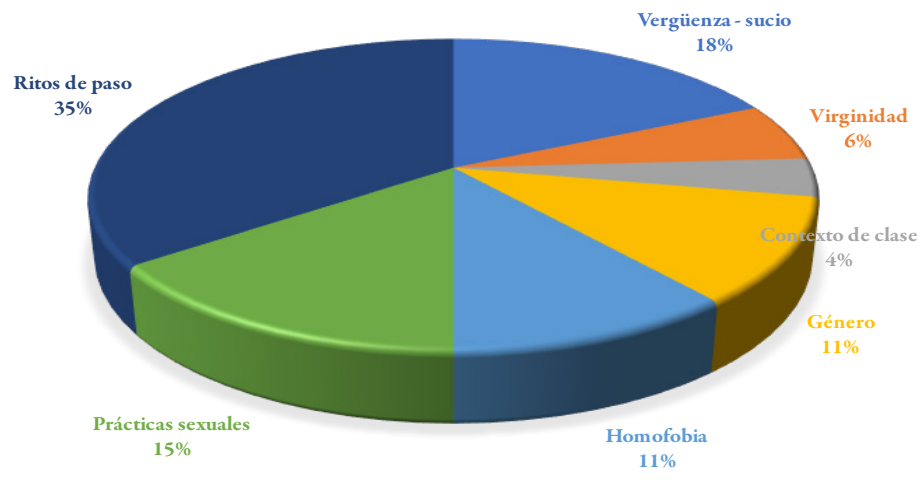

Fuente: Elaboración propia a partir de las entrevistas realizadas. 
Mar Venegas. Profesora Titular de Sociología de la Universidad de Granada, España, de la que es actualmente Subdirectora. Doctora en Sociología, con Mención de Doctorado Europeo, y Experta en Género e Igualdad de Oportunidades por esta universidad. Líneas de investigación: sociología de la educación, estudios feministas y de género, diversidad e inclusión social. Publicaciones recientes: Venegas, Mar (2018), "El romance adolescente. Un análisis sociológico de la política afectivosexual en la adolescencia”, en Papers. Revista de Sociología, vol. 103, núm. 2. Disponible en: https://papers.uab. cat/article/view/v103-n2-venegas; Issó, David, Becerril, Diego, MartínLagos, Lola y Venegas, Mar (2019), “Ojalá mi madre hubiera hecho por mí lo que yo estoy haciendo por mi hija. La evolución de la participación de las familias en la escuela pública en España", en Sociología. Problemas e Prácticas, núm. 89. Disponible en https://journals.openedition.org/spp/5413; Venegas, Mar, Villar-Aguilés, Alícia, Santos, Sofía A. (2020), "Sex and gender equality policies in education in Southern European Societies: the three cases of Andalusia and the Valencian Community (Spain) and Portugal", en Revista Española de Sociología (RES), vol. 29, núm. 1. Disponible en: https://recyt.fecyt.es/index.php/res/article/view/74730 